\title{
Percutaneous edge-to-edge mitral valve repair for secondary mitral regurgitation: perspectives on COAPT and MITRA-FR trials
}

\author{
Nirvik Pal, Mark Nelson, John Butterworth \\ Department of Anesthesiology, Virginia Commonwealth University, Virginia, VA 23298-0541, USA.
}

Correspondence to: Nirvik Pal, Assistant Professor in Anesthesiology, Division of Cardiothoracic Anesthesiology, Departmen of Anesthesiology, 1250 East Marshall St, 5th Floor, PO Box 980541, Richmond, Virginia, VA 23298-0541, USA.

E-mail: nirvik.pal@vcuhealth.org

How to cite this article: Pal N, Nelson M, Butterworth J. Percutaneous edge-to-edge mitral valve repair for secondary mitral regurgitation: perspectives on COAPT and MITRA-FR trials. Vesse/P/us2019;3:39. http://dx.doi.org/10.20517/2574-1209.2019.24

Received: 17 Jul 2019 First Decision: 22 Aug 2019 Revised: 20 Sep 2019 Accepted: 24 Sep 2019 Published: 12 Dec 2019

Science Editor: Mario F. L. Gaudino Copy Editor: Jing-Wen Zhang Production Editor: Jing Yu

Mitral valve regurgitation (MR) is classified as primary or secondary depending upon pathophysiology. Primary MR arises from degenerative disease of the valve leaflets whereas secondary MR is due to dilatation of left ventricle leading to distortion of valve architecture ${ }^{[1]}$. Therapy for primary MR is established: valve repair (surgery or percutaneous), or valve replacement when repair is not possible ${ }^{[2,3]}$. In contrast, therapy for secondary MR is still a matter of debate ${ }^{[4]}$. It is universally agreed that treatment for left ventricular dilatation is foremost by guideline directed medical therapy (GDMT) and cardiac resynchronization therapy ${ }^{[3]}$. Whether to treat the MR mechanically constitutes the dilemma. This intriguing conundrum becomes even more interesting with publication of two similar trials with opposite results $^{[5,6]}$. If reproducibility is the foundation of settled new knowledge, then why did these two large randomized controlled trials have seemingly irreconcilable results?

Investigators of the COAPT (Cardiovascular Outcomes Assessment of the MitraClip Percutaneous Therapy for Heart Failure Patients with Functional Mitral Regurgitation, USA) ${ }^{[6]}$ and the MITRA-FR (Percutaneous Repair with the MitraClip Device for Severe Functional/Secondary Mitral Regurgitation, France $)^{[5]}$ trials hoped to seek whether secondary MR was simply a marker of a diseased and dilated left ventricle or separately contributed to patient mortality and therapy via edge-to-edge percutaneous repair of mitral valve (MV) (MitraClip ${ }^{\circledR}$ ) would be efficacious. Incidentally, results of the MITRA-FR trial support the former assertion while those of COAPT trial seem to corroborate the later. 
Table 1. Comparison between MITRA-FR and COAPT trials

\begin{tabular}{|c|c|c|}
\hline & MITRA-FR & COAPT \\
\hline Enrolled patients & 307 & 614 \\
\hline Duration of follow-up & 1-year & 2-year \\
\hline HF medications & $\begin{array}{l}\text { Appropriate; titrations permitted after } \\
\text { randomization }\end{array}$ & Maximized before randomizations \\
\hline Severe MR (criteria by EROA) & $>0.2 \mathrm{~cm}^{2}$ & $>0.3 \mathrm{~cm}^{2}$ \\
\hline LV dilatation (LVESD) & Unspecified & $>70 \mathrm{~mm}$ excluded \\
\hline Mean EROA at baseline & $0.31 \mathrm{~cm}^{2}\left(52 \%<0.3 \mathrm{~cm}^{2}\right)$ & $0.41\left(41 \% \geq 0.4 \mathrm{~cm}^{2}\right)$ \\
\hline Mean LVEDV at baseline & $252 \mathrm{~mL}$ & $192 \mathrm{~mL}$ \\
\hline NYHA stage III-IV & $65 \%$ & $58 \%$ \\
\hline Post-procedure MR grade $3+/ 4+$ & $<10 \%$ & $<10 \%$ \\
\hline Complications (procedural) & $14.6 \%$ & $8.5 \%$ \\
\hline MR at 1-year ( $\geq 2$ grade) & $49.5 \%$ & $31.0 \%$ \\
\hline HF hospitalizations at 1-year & $49 \%$ & $38 \%$ \\
\hline LV volume change & None & Decreased \\
\hline Mortality (30-day) & $3.3 \%$ & $2.3 \%$ \\
\hline Mortality (1-year) & $24 \%$ & $19 \%$ \\
\hline Hazard ratio for all-cause mortality $(95 \% \mathrm{Cl})$ & 1.11 (0.69-1.77) (No benefit) & $0.62(0.46-0.82)$ (38\% risk reduction) \\
\hline
\end{tabular}

HF: heart failure; LV: left ventricular; MR: mitral valve regurgitation; EROA: effective regurgitant orifice area; LVESD: left ventricular end systolic diameter; LVEDV: left ventricular end diastolic volume; MITRA-FR: percutaneous repair with the mitraclip device for severe functional/secondary mitral regurgitation; COAPT: cardiovascular outcomes assessment of the mitraclip percutaneous therapy for heart failure patients with functional mitral regurgitation

\section{COMPARE AND CONTRAST}

The New England Journal of Medicine published articles detailing the results of these two trials with contradictory conclusions in the same 2018 issue. What followed were multiple viewpoints and reviews comparing and analyzing the trials apparent opposite results ${ }^{[7-10]}$. We intend to review several of these interpretations as well as assess the methodologic and study population differences between these two studies [Table 1].

Underlying an informed analysis of MITRA-FR and COAPT trials is a new concept: "disproportionate"s] or "tertiary" $\mathrm{MR}^{[7]}$. As explained by Packer et al. ${ }^{[8]} \mathrm{MR}$ severity may or may not be proportional to left ventricular (LV) dilation, and also may or may not contribute to the disease process. In some patients the MR may become exaggerated or "out of proportion" (disproportionate MR) to their LV dilatation. In these patients with disproportionately increased MR, the regurgitation may represent a causative agent for (or contributor to) worsening heart failure (HF), rather than an innocent "bystander". In retrospect, by its inherent study design and inclusion criteria, the MITRA-FR trial enrolled all patients with secondary $\mathrm{MR}$, irrespective of "proportionality" in degree of MR. In contrast, COAPT trial used more stringent selection criteria and included a greater percentage of patients with "disproportionate" MR. MITRA-FR trial enrolled an "all comers" patient population and was unable to show any benefit of percutaneous edgeto-edge repair of MV. Conversely, the COAPT trial was able to show remarkable clinical benefit of the procedure. Put another way, these two studies enrolled different populations using different study protocols and we are not surprised that the results were also different.

\section{SPECIFIC SIMILARITIES AND DIFFERENCES}

What were the differences in the COAPT and MITRA-FR study designs? In MITRA-FR, included subjects were required to have at least one prior HF hospitalization within 12 months and MR severity with effective regurgitant orifice area $(\mathrm{EROA})>20 \mathrm{~mm}^{2}$ or $\mathrm{MR}$ regurgitation volume $(\mathrm{RV})>30 \mathrm{~mL}$. B-natriuretic peptide (BNP) and left ventricular end systolic diameter (LVESD) were not specified. The primary endpoint was a composite of unplanned hospitalization for HF at 12 months or all-cause death. Whereas in COAPT, 
study subjects were required to have at least one of three criteria: at least one hospitalization for HF within 12 months, BNP $>300 \mathrm{pg} / \mathrm{mL}$ or NT-proBNP $>1500 \mathrm{pg} / \mathrm{mL}$, or MR severity with EROA $>30 \mathrm{~mm}^{2}$ or RV $>45 \mathrm{~mL}$. The primary outcome was HF hospitalizations within 24 months of follow-up ${ }^{[10]}$. As a result, in MITRA-FR trial versus COAPT trial the average NT-proBNP, 12 months prior annual hospitalizations and LVEDV were approximately $3349 \mathrm{ng} / \mathrm{L} v$ s. $5558 \mathrm{pg} / \mathrm{mL} ; 100 \%$ vs. $57 \%$; and $135 \mathrm{~mL} / \mathrm{m}^{2} v s .101 \mathrm{~mL} / \mathrm{m}^{2}$ respectively. Nishimura et al. ${ }^{[11]}$ in their accompanying editorial point out several features that could explain the differences: first, the strategy of medical management before and after enrollment differed. In COAPT trial, maximal HF treatment took place solely prior to randomization, therefore identifying patients truly refractory to medical therapy. After enrollment, further adjustments to medical therapy were not allowed. In MITRA-FR trial, medical therapy modifications were permitted before and after randomization. Second, the baseline valvular and ventricular characteristics were different. In COAPT, the MR was more severe compared to MITRA-FR (average EROA, $41 \mathrm{~mm}^{2}$ vs. $31 \mathrm{~mm}^{2}$ ) but with a smaller LV size [left ventricular end diastolic volume index (LVEDVI) $101 \mathrm{~mL} / \mathrm{m}^{2} v s .135 \mathrm{~mL} / \mathrm{m}^{2}$ \& left ventricular end diastolic diameter, $62 \mathrm{~mm} v s .69 \mathrm{~mm}$ ]. Third, procedural skill may have contributed, as COAPT had more patients with multiple clips than MITRA-FR and possibly more experienced proceduralists since the study took place at a later date. Primary endpoint in COAPT trial which was HF hospitalization within 24 months of procedure was $35.8 \%$ in intervention $v s .67 .9 \%$ in control arm $(P<0.001)$. Primary endpoint in MITRAFR, which was death or HF hospitalization at 12 months was $54.6 \%$ in intervention group vs. $51.3 \%$ in control $(P=0.53)$.

After the studies and editorial were published, readers from all over the world submitted queries. Regarding COAPT trial, Crestanello et al. ${ }^{[12]}$ were curious about the echocardiographic methodology in calculation of EROA as for them (Simpson's method of discs) it would generate impractical numbers for both regurgitant volume and stroke volume. The authors explain this discrepancy due to difference in echocardiographic measurement techniques (Simpson's vs. Doppler) ${ }^{[13]}$. To us, this emphasizes the inherent limitations of echocardiography, and questions validity of conclusion based on semiquantitative echocardiographic estimates. Drake et al. ${ }^{[14]}$ speculate that detailed knowledge of the preoperative transesophageal echocardiography could have introduced a bias to the interventionists' in COAPT trial leading to better outcomes. The authors agree with this observation emphasizing the value of preoperative advanced imaging and thus its use is representative of current medical practice and therefore should be included in the study protocol. Garbi et al. ${ }^{[15]}$ recognize that the "disproportionate" ${ }^{\text {p[ }}$ or "tertiary" $\mathrm{MR}^{[7]}$ along with reduced left atrial pressure observed in COAPT patients, both may have contributed to improved symptoms post-procedurally which the authors agree with as a possibility. Despite that the COAPT trial was company sponsored (Kalavrouziotis et al ${ }^{[16]}$ ), the authors posit an independence of medical decision making.

Godino et al. ${ }^{[17]}$ expressed concerns about the very frequent (> 20\%) occurrence of mortality in both intervention and control groups in MITRA-FR trial. In response, the authors compared mortality at oneyear in both the MITRA-FR and COAPT trials and found relatively similar mortality at one year ${ }^{[18]}$. The study investigators also explain that the other major heart failure trials with lesser one year mortality were not comparable due to differences in study designs. For example, the PARADIGM-HF trial explicitly excluded "all hemodynamically significant mitral disease". Goliasch et al ${ }^{[19]}$ point out that the differing definition of severe MR between the COAPT (EROA $0.3 \mathrm{~cm}^{2}$ ) and the MITRA-FR (EROA $0.2 \mathrm{~cm}^{2}$ ) trials, reflecting American vs. European guidelines, could have contributed to the difference in results as well. Nevertheless, a sub-analysis controlling for EROA did not change the results. Silverio et al ${ }^{[20]}$ posit that the lack of benefit observed in the MITRA FR trial could be due to relatively nonrestrictive study centereligibility criteria, and inexperience on part of the proceduralist. The MITRA-FR authors point out that, despite the fact that they had only a five procedure per center minimal requirement, the COAPT had three subjects per center for active enrollment. Therefore, although COAPT trial ended up with more patients 
per center, but criteria for eligibility was not much different than MITRA-FR trial. Lastly, as previously noted, the MITRA-FR authors do not deny that they may have included patients with heart failure too advanced to derive benefit ${ }^{[18]}$.

Intensive analysis of these two trials have led most practitioners to agree that the trials are "complimentary rather than contradictory" (Tang et al. ${ }^{[21]}$, Carabello et al. ${ }^{[7]}$ ). Whether the diagnosis is "disproportionate MR" (Packer et al.$^{[8]}$, Praz et al. ${ }^{[9]}$ ) or "tertiary $\mathrm{MR}^{\text {") }}$, consensus appears to be that patients with the combination of severe secondary MR (EROA $\left.>0.3 \mathrm{~cm}^{2}\right)$ with no more than moderate LV dilatation (LVEDVI $<96 \mathrm{~mL} / \mathrm{m}^{2}$ ) potentially will benefit from percutaneous edge-to-edge repair (MitraClip ${ }^{\circledR}$ ) procedure after optimal medical therapy, as shown in COAPT trail.

Furthermore, as pointed out by Praz et al ${ }^{[9]}$, these two trials lead to the following conclusions regarding percutaneous therapy for secondary MR:

1. Extreme LV dilatation with less severe secondary MR (no benefit: MITRA-FR trial).

2. Moderate LV dilatation with more severe secondary MR (benefit: COAPT trial).

3. Extreme LV dilatation with more severe secondary MR (unknown benefit).

\section{SURGICAL PERSPECTIVE}

The American Association for Thoracic Surgery in 2016 revised their guideline recommendation for severe secondary MR to MV replacement rather than repair for patients with LVEDD $>65 \mathrm{~mm}^{[1]}$. Surgical correction of secondary MR has been a topic of great interest and a matter of equipoise ${ }^{[4]}$. The challenge has been that surgical anatomic correction has not necessarily translated into a lasting clinical benefit ${ }^{[2]}$. Conceptually, the basis for surgical correction has been to produce a normal architecture, most often with placement of a mitral annuloplasty ring. Edge-to-edge percutaneous technique without any reinforcement with annular ring, seems unconvincing. Badhwar et al. ${ }^{[23]}$ posit that while MV replacement is best for symptom resolution, valve repair still has its "niche" role in pathoanatomically suitable patients with secondary MR. They suggest an entire pathoanatomic classification based on: (1) annular dilatation; (2) ejection fraction; and (3) leaflet tethering. Based on this they suggest "low surgical risk patients" may undergo CABG + mitral valve repair or replacement whereas "high surgical risk" may have catheter based transmitral valve replacement or repair. As a caveat, this hypothesis has not been tested yet in prospective randomized clinical trials. Definitive conclusions regarding the relative efficacy of other techniques for secondary MR including percutaneous neo-chord, percutaneous annuloplasty ring ${ }^{[24]}$, and percutaneous MV replacement await appropriate clinical studies. In the light of these evolving techniques. Future guidelines may increasingly rely on assessment of surgical risk, likelihood of successful anatomic correction and clinical benefit and feasibility.

\section{CONCLUSION AND FUTURE STUDIES}

Percutaneous edge-to-edge mitral valve repair for mitral regurgitation has been practiced in Europe since approved by the European Commission in 2008. It is estimated that $65 \%$ of percutaneous repair for MV in Europe are for secondary MR. In contrast, in North America, the United States Food and Drug Association approved this procedure in 2013 only for primary (degenerative) mitral regurgitation, consequently about $80 \%$ of all MitraClip procedures in US are for primary MR. Thus, Europeans overall have a much broader experience in MitraClip ${ }^{\circledR}$ procedures. This may be reflected in the approach taken by the MITRAFR authors, namely that percutaneous MV repair would be more readily offered on a less stringent basis compared to the more medically refractory subjects included in the COAPT. For certain, percutaneous edge-to-edge repair is not for every patient with secondary MR. That said, there is one specific subset of patients that did show benefit. Identification of these patients and successful percutaneous valve repair will require meticulous medical optimization of heart failure, careful echocardiographic measurements and a 
skilled interventional cardiologist. Once completed, RESHAPE-HF-2 (NCT02444338) should better define the role of transcatheter edge-to-edge repair of secondary MR. Finally, we eagerly await longer term studies that assess repair durability, compare different clip designs, and refine optimal medical management of patients before and after percutaneous repairs.

\section{DECLARATIONS}

\section{Acknowledgments}

Sincere gratitude to Dr. Zachary Gertz, Director Interventional Cardiology, VCU Health System, Richmond, Virginia for his inputs on revising the manuscript.

\section{Authors' contributions}

Design, content, datacollection, literature search, discussion, inferences: Pal N

Design, content, discussion, inferences: Nelson M

Content, discussion, inferences, language ammendments: Butterworth J

\section{Availability of data and materials}

Not applicable.

\section{Financial support and sponsorship}

None.

\section{Conflicts of interest}

All authors declared that there are no conflicts of interest.

\section{Ethical approval and consent to participate}

Not applicable.

\section{Consent for publication}

Not applicable.

\section{Copyright}

(c) The Author(s) 2019.

\section{REFERENCES}

1. Kron IL, Acker MA, Adams DH, Ailawadi G, Bolling SF, et al; American Association for Thoracic Surgery Ischemic Mitral Regurgitation Consensus Guidelines Writing Committee. 2015 The American Association for Thoracic Surgery Consensus Guidelines: Ischemic mitral valve regurgitation. J Thorac Cardiovasc Surg 2016;151:940-56.

2. Feldman T, Foster E, Glower DD, Kar Saibal, Rinaldi MJ, et al. Percutaneous repair or surgery for mitral regurgitation. N Engl J Med 2011;364:1395-406.

3. Nishimura RA, Otto CM, Bonow RO, Carabello BA, Erwin JP, et al. 2017 AHA/ACC focused update of the 2014 AHA/ACC guideline for the management of patients with valvular heart disease: a report of the American College of Cardiology/American Heart Association task force on clinical practice guidelines. Circulation 2017;70:252-89.

4. Goldstein D, Moskowitz AJ, Gelijns AC, Ailawadi G, Parides MK, et al. Two-year outcomes of surgical treatment of severe ischemic mitral regurgitation. N Engl J Med 2016;374:344-53.

5. Obadia JF, Messika-Zeitoun D, Leurent G, Iung B, Bonnet G, et al. Percutaneous repair or medical treatment for secondary mitral regurgitation. N Engl J Med 2018;379:2297-306.

6. Stone GW, Lindenfeld J, Abraham WT, Kar S, Lim DS, et al. Transcatheter mitral-valve repair in patients with heart failure. N Engl J Med 2018;379:2307-18.

7. Carabello BA. MitraClip and tertiary mitral regurgitation-mitral regurgitation gets curiouser and curiouser. JAMA Cardiol 2019;4:307-8.

8. Packer M. When cardiovascular trials collide. Eur Heart J 2019;40:501-4. 
9. Praz F, Grasso C, Taramasso M, Baumbach A, Piazza N, et al. Mitral regurgitation in heart failure: time for a rethink. Eur Heart J 2019;40:2189-93.

10. Senni M, Adamo M, Metra M, Alfieri O, Vahanian A. Treatment of functional mitral regurgitation in chronic heart failure: can we get a "proof of concept" from the MITRA-FR and COAPT trials? Eur J Heart Fail 2019;21:852-61.

11. Nishimura RA, Bonow RO. Percutaneous repair of secondary mitral regurgitation - a tale of two trials. N Engl J Med 2018;379:2374-6.

12. Crestanello JA, Oh JK, Schaff HV. Transcatheter mitral-valve repair in patients with heart failure. N Engl J Med 2019;380:1978.

13. Stone GW, Weissman NJ, Mack MJ, COAPT Investigators. Transcatheter mitral-valve repair in patients with heart failure. Reply. N Engl J Med 2019;380:1980-1.

14. Drake DH, Zimmerman KG, Sidebotham DA. Transcatheter mitral-valve repair in patients with heart failure. N Engl J Med 2019;380:1978-9.

15. Garbi M, Lancellotti P. Transcatheter mitral-valve repair in patients with heart failure. N Engl J Med 2019;380:1979.

16. Kalavrouziotis D, Voisine P, Mohammadi S. Transcatheter mitral-valve repair in patients with heart failure. N Engl J Med 2019;380:1979-80.

17. Godino C, Munafo A, Margonato A. Percutaneous repair for secondary mitral regurgitation. N Engl J Med 2019;380:1975-6.

18. Grinberg D, Obadia JF, Iung B. Percutaneous repair for secondary mitral regurgitation. Reply. N Engl J Med 2019;380:1977-8.

19. Goliasch G, Bartko PE, Hulsmann M. Percutaneous repair for secondary mitral regurgitation. N Engl J Med 2019;380:1976.

20. Silverio A, Baldi C, Citro R. Percutaneous repair for secondary mitral regurgitation. N Engl J Med 2019;380:1976-7.

21. Tang GHL, Verma S, Bhatt DL. Two randomized clinical trials on the treatment of secondary mitral regurgitation-contradictory or complementary? JAMA Cardiol 2019;4:311-3.

22. Acker MA, Parides MK, Perrault LP, Moskowitz AJ, Gelijns AC, et al. Mitral-valve repair versus replacement for severe ischemic mitral regurgitation. N Engl J Med 2014;370:23-32.

23. Badhwar V, Alkhouli M, Mack MJ, Thourani VH, Ailawadi G. A pathoanatomic approach to secondary functional mitral regurgitation: evaluating the evidence. J Thorac Cardiovasc Surg 2019;158:76-81.

24. Tozzi P. The future of functional mitral regurgitation treatment. Eur Heart J 2019;40:2215-7. 